\title{
Il Diritto Cambiario Brasiliano e la Legge Uniforme di Ginevra*
}

\author{
Waldemar Ferreira \\ Professore Emérito nella Facolta di \\ Diritto dell'Universita di San Paolo.
}

1. Non senza orgoglio scrisse il Casaregi che fu "il nuovo contratto del cambio trovato daí nostri concittadini della parte Guelfa per ritirare senza spesa, e pericolo le loro sostanze dalla patria in Lione, ove cacciati dalla parte contraria dai Ghibellini, s'erano rifugiati".

Questa affermata invenzione della cambiale, secondo GoldSGHMIDT, "é una fiaba da balie, e le si fa già troppo onore ricordandola". Può esserlo, o no; ma in verità in quei tempi e da molti anni il contratto di cambio rispondeva ad un'applicazione del negozio di rimessa di denaro da piazza a piazza, da piazza a fiera, o da fiera a fiera.

Al luogo della fiera, proprio il GoLdschmidt lo ha detto, "dovevano dirigersi le somme, che i compratori della fiera non volevano portare essi stessi, oppure dovevano riscuotere colà da debitori; dal luogo della fiera doveva rimettersi il prezzo ricavato da merci vendute, e così pure l'ammontare di pagamenti ricevuti per altro titolo, ad altre piazze, a un altro luogo di fiera o alla patria del venditore, e così via" 1

(*) Apud Scritti Giuridici in Memoria di Lorenzo Mossa, Casa Editrice Dottor Antonio Milani, Pádua, 1959; Rivista del Diritto Commerciale, Milão, 1959,Casa Editrice Dr. Francesco Vallardi, Ano LVII (1959), Parte 1, págs. 1 a 12.

1. Levin Goldschmitd, Storia Universale del Diritto Commerciale, Trad. di Vittorio Pouchain \& Antonio Scialoja, Torino, 1913, Unione Tipografico-Editrice Torinese, p. 315. 
Molto si era già discusso in quell'epoca sulla natura giuridica dell'operazione di cambio.

L'aveva individuata il CASAREgI in tutta la sua complessità come contratto di cambio "obliquo, ed indiretto, in cui si occultano tre contratti, cioé il mutuo, il mandato, ed il cambio: s'occulta il mutuo perché il creditore impresta il denaro al debitore per le sue indigenze; s'occulta il mandato, perché non volendo il creditore tenere ozioso il denaro, il debitore lo costituisce procuratore a prendere l'équivalente somma da altri, o da se medesimo, e girarla a danno suo per le fiere, oppure egli stesso promette di farne la circolazione: s'occulta finalmente il cambio, perché il creditore, o il debitore dovrebbe cambiare; e se il creditore cambia, lucra i cambi, e se non cambia gli perde; all'incontro se il debitore non cambia, è tenuto ai cambi, non come cambi, perché non ha cambiato, ma come interessi, perché doveva cambiare, ancorchè il creditore si fosse riservata la facoltà di cambiare, ed ancor esso non avesse cambiato, e questo cambio é approvato come gli antecedenti dalla nostra Santa Chiesa" 2 .

Da tale esposizione si rileva che il contratto di cambio non si riputava come di mutuo, per ottenere l'approvazione dalla Santa Chiesa; ma non ha il CaSAREgi considerato l'ipotesi da lui stesso segnalata in altro punto del suo discorso, di trattarsi, nel contratto di cambio, di una vendita, in ragione della possibilità di una negoziazione del "denaro come mercanzia"

Non é quel contratto, come egli stesso ha affermato, altro che "una compra del denaro assente, cambiandose col presente", non ostante che "il denaro sia stato propriamente per prezzo delle cose vendibile", tanto piú che "considerato come corpo ancor esso si vende, e si permuta".

Questo é stato anche il pensiero di un commercialista brasiliano del 1798 - il José da Silva Lisboa, nei Princi-

2. Joseph LaUrentil Mariae de CASARegis, Discursus Legales de Commercio, Florentiae, MDCCXXXIX, Typis, et Sumptibus Bernardi Paperini, Tom. IV, Disc. CCXVIII, §§ $1 / 3$. 
pii di Diritto Mercantile e Leggi di Marina, che l'AscaRELLI ha detto essere il "piú acuto e moderno trattato di diritto mercantile dai fini del Secolo XVIII e principi del Secolo XIX" 3.

Ritenendo il contratto di cambio un contratto di diritto delle genti e riconoscendogli carattere specifico per la singolarità dei suoi effetti, il Silva Lisboa ha sostenuto che esso presentava la maggiore analogia con la compravendita, a punto di regolarsi per le disposizioni del diritto civile, con le modificazione introdotte per il commercio, risultanti dall'efficacia e rapidità dei mezzi essenziali alla letcera di cambio.

2. I contratti di cambio furono in verità approvati nel 1570 con la Bolla In eam pro nostro Pastorali officio dal Pontefice PIo IV. Ma non fu arbitrariamente che la Chiesa dichiarò la diversità di luogo come requisito essenziale dell'affare di cambio. Cambiorum usus quem necessitas, \& pubblica utilitas induxit. Come ha osservato a questo proposito il Goldschmidt, la Chiesa "invece non ostante le proprie particolari preoccupazioni ha riconosciuto il vero affare di cambio, ma all'affare locale, che secondo l'idee commerciali e il diritto (laico) del medioevo non poteva essere "affare di cambio", ha rifiutato il riconoscimento di affare cambiario (lecito), che era agognato per diversi motivi".

Si riteneva il negozio di cambio locale pecato di usura, per la possibilità esistente di simulazione del prestito usurario.

Ecco perché nel Portogallo, fedele alla dottrina della Chiesa, alcuni anni dopo, le Ordinanze del Re FILIPpo II, del 1603, hanno regolato il contratto di cambio e la cam-

3. Tullio Ascarelli, $A$ evolução do Direito Comercial. $A$ unificação do Direito das Obrigações, na Revista de Direito Comercial, Rio de Janeiro, 1941, v. II, p. 34, n. 3; José DA Silva LISBOA, Principios de Direito Mercantil e Leis de Marinha para uso da Mocidade Portuguêsa dedicada ao Comércio, Lisboa, 1798 a 1804, Regia Oficina. Tipografica, tômo IV, Tratado IV, Las Letras de Câmbio, cap. II. 
biale nel titolo "dei contratti usurari" Era antico davvero in quel paese l'uso delle lettere di cambio; ma le sue Ordinanze determinavano che le persone che dessero il loro denaro al cambio o lo pagassero, non facessero differenza alcuna tra il suo impiego in contante o in lettera o vaglia cambiario (livrança), ottenendo interessi maggiori nel pagamento in moneta di quelli che comunemente si pagavano nella piazza per il cambio per mezzo dei vaglia.

A mantenere in vita questa tesi, le Ordinanze avevano stabilito che era lecito soltanto il guadagno di denaro "in qualsiasi caso di cambio da un Regno o luogo ad un altro". Ma c'era di piú: dichiaravano "lecito e vero il cambio" soltanto quando si consegnava un certo importo in denaro in un luogo affinché in un altro si pagasse un poco meno. Sarebbe la differenza, il corrispettivo l'aggio. Questo sconto si giustificava ed era permesso dal diritto in ragione delle spese che $i$ mercanti residenti nel luogo dovevano fare per la manutenzione dei loro cambi.

La forza della religiosità medioevale perdurava nelle costruzioni giuridiche e si fatta sentire nel contratto di cambio.

Se in quei tempi l'istituto della rappresentanza, peculiare del diritto romano, secondo il quale alteri stipulare non potest, non era predominante, tuttavia si verificava una rinascita della rappresentanza, affermata dal diritto canonico nella regola secondo la quale qui facit per alium est proinde ac si faciat per se ipsum.

Senza l'adozione di questo principio non era possibile l'esecuzione del contratto di cambio: il cambiatore per eseguirlo doveva essere titolare di un diritto di credito contro il banchiere o mercante che lo rappresentava o che era da esso rappresentato.

Di molta acutezza l'osservazione del GoLdschmidT secondo la quale si troverebbe nelle dichiarazioni scritte di debito una clausola che é la chiave della storia, finora misteriosa, dello sviluppo della cambiale - la clausola all' ordine passiva. Ed infatti "come con la clausola all'ordine attiva colui che emette un titolo fonda il diritto in questo 
formolato, non soltanto per il prenditore nominato nel titolo, ma anche per l'ordine di lui, cioé per colui, che sará designato dal prenditore nominato (tibi vel tuo certo nuntio, mandato, cui mandaveris, ordinaveris, etc.) così l'emittente può inoltre dichiarare, che egli si riserva di pagare per mezzo di un altro la somma promessa: per me vel per meum nuncium, missum e simili. Quest'altro egli poteva nominarlo subito, o almeno designarlo esattamente (per es. per meos socios), oppure poteva frattanto lasciarlo affatto indeterminato".

Sebbene il diritto al denaro avuto come mercanzia sia molto piú esatamente configurabile como diritto di credito che come diritto di proprietà nel significato comune di questa espressione, la sua trasmissione si é praticata da tempo immemorabile. Ma poiché il diritto di credito si realizzava solo mediante un documento notarile o giudiziario - la carta, questo documento aveva un valore patrimoniale. Il trasferimento di quel diritto non era possibile senza la tradizione del documento.

Quando la pratica degli affari sospese quel documento notarile, sostituito dalla lettera di cambio, s'affermo il principio della cessione di credito.

Il contratto di cambio si é arrestato nelle linee della sua evoluzione fino a trasformarsi in un titolo di credito per effetto dell'introduzione della girata nel suo meccanismo e nella sua struttura giuridica: si é fatta una revoluzione nell'ambito del diritto cambiario.

Che gli svolgimenti di cui si é fatto cenno siano intimamente legati alla liberazione del contratto di cambio dalla condanna per usura, per il pungolo delle necessità commerciali, é ovvio.

L'esigenza benefica e legittima di reprimire l'usura ha impedito, secondo il LEVY-BruHL, la naturale evoluzione di molti istituzioni commerciali. I commercialisti si sono

4. Levin Goldschmidt, Storia Universale del Diritto Commerciale, Trad. di Vittorio Pouchain \& Antonio Scialoja, Torino, 1913, Unione Tipografico-Editrice Torinese, p. 307. 
appellati a tutti i mezzi della loro dialettica per dimostrare che il contratto di cambio non era un mutuo, ma una vendita, almeno un atto giuridico sui generis che non poteva essere considerato come usurario. La lettera di cambio per se stessa non era libera delle sanzioni canoniche o teologiche, salvo che si presentasse come mezzo di una trasmissione da luogo a luogo: la distantia loci era la sua condizione primordiale ${ }^{5}$.

Questa era la posizione della cambiale prima del Secolo xvin, durante il quale l'autorità secolare ha favorito l'utilizzazione di quel titolo nelle relazioni internazionali. Ma in Francia già si erano create condizioni d'ordine economico per la sua consacrazione legislativa.

3. Bene ha detto il CaLasso che strettamente legata a queste nuove concezioni é una istituzione che nella vita commerciale del rinascimento era destinata ad assumere una importanza enorme - il titolo al portatore; e se é vero che la nascita di questo fu lentamente preparata nei secoli dell'alto medio evo attraverso il processo di incorporazione del diritto nella carta che lo documentava, é peró innegabile che una funzione cosi importante nel vortice degli affari, fin quasi a rappresentarne il simbolo, esso potette raggiungere solo nella nuova economia monetaria ${ }^{6}$.

La lettera di cambio così preparata per opera dei mercanti nel tramonto del medio evo appunto attraverso la incorporazione del diritto nel titolo che lo documentava, raggiunse una formazione definitiva in Francia nel 1673, nell'Ordonnance sur le Commerce.

Il diritto cambiario della rivoluzione francese, come scrisse il Mossa, fu erede della tradizione che si incarnava massimamente nell'ordinanza del commercio. La rivoluzione aveva abbattuto privilegi e creato un nuovo ordine

5. HENRI LeVY-BRuhL, Histoire de la Lettre de Change aux XVII et XVIII, Siècles, Paris, 1933, Recueil Sirey, p. 22.

6. Francesco Calasso, Introduzione al Diritto Comune, Milano, 1951, Dott. A. Giuffré, editore, p. 151. 
giuridico, ma il diritto cambiario si era silenziosamente conservato, e persino qualche rara voce di rinnovamento era svanita ".

Restò così segnalata quell'ordinanza francese, che preparò l'avvenimento del Code de Commerce del 1707.

Questo codice, come scrisse il Fremery, ha creato il vero diritto commerciale, opera lenta e sucessiva dei mercanti, le cui fonti si trovano nei monumenti delle loro consuetudini. Da quel momento in poi, il diritto commerciale, che doveva essere semplice, grande, universale come il commercio che lo aveva prodotto, si é spezzato, divenendo, volta per volta, diritto commerciale francese, diritto commerciale inglese, diritto commerciale spagnuolo ${ }^{8}$.

Ed anche diritto commerciale brasiliano.

Proclamata nel 1822 la separazione del Brasile dalla sfera coloniale del Portogallo, la trasformazione politica del paese doveva pur produrre la trasformazione della sua struttura giuridica. Non cessarono tuttavia di vigorare le leggi della metropoli, soprattuto quelle di carattere privato, anche nel momento stesso del avvento politico della independenza della nazione.

Poichè il diritto, espressione del grado di civilità dei popoli, trasforma $i$ suoi istituti in consonanza ai bisogni ed alle esigenze della vita nazionale, giurata la Costituzione Politica dell'Impero del Brasile nel 1824, l'ordinamento giuridico rivocó come prima legge nel 1830 il Codice Criminale.

Il Codice Commerciale fu promulgato nel 1850.

Non é questo il momento di fare l'elogio di questo codice, in buona parte ancora in vigore, o la sua elegia; ma é da considerare che $i$ suoi elaboratori non praticarono il peccato di fare sfoggio dell'originalità. Essi raccolsero dai codice commerciali allora in vigore quanto di

7. LoRenzo Mossa, Trattato della Cambiale, Padova, $1956,3 .^{\circ} \mathrm{ed}$. Cedam - Casa Editrice Dott. Antonio Milani, p. 1, n. 1.

8. A. FRemery, Etudes de Droit Commercial, ou du Droit Fondé par la Coutume Universelle des Commerçants, Paris, 1833, Alex-Gobelet, éditeur, p. 19. 
piú eletto vi era nella scienza giuridica compatibile con la tradizione ed i bisogni della pratica commerciale del paese; e l'opera della codificazione sotto questo riguardo ed anche per il suo carattere organico ha resistito in parte all'azione del tempo: é piú che centenario il codice brasiliano.

4. Circa la dottrina regolatrice della cambiale, il Codice Commerciale dell'Impero del Brasile ha adottato quella predominante in quei tempi; ma non ha perduto il senso tradizionale attraverso in cui la cambiale era stata a lungo usata nel commercio ed era stata interpretata dai tribunali.

Si tripartiva la cambiale nella lettera di cambio, nella lettera di terra e nella nota promissoria, ossia nel "pagheró", o vaglia cambiario.

La lettera di cambio doveva contenere piú o meno i requisiti del titolo odierno ed esprimere la valuta ricevuta, con la specificazione se in moneta, ed il suo ammontare, se in merce, se in conto od in qualunque altro modo, il che era condizione intrinseca del titolo. Essa certamente si riputava uno strumento di credito, nonostante fosse concepita quale convenzione obbligante l'uno dei contraenti a far pagare una certa somma in un luogo determinato, per un valore promesso o dato in altro luogo.

Cosi strutturata, la lettera di cambio era emessa dall'una all'altra delle Provincie dell'Impero; ed in ciò consisteva l'elemento differenziale tra la lettera di cambio e la lettera di terra: questa era emessa ed accettata nella stessa Provincia.

La nota promissória o vaglia cambiario, come titolo contenente la promessa di pagamento di una somma determinata, s'equiparava alla lettera di terra, e così pure gli scritti particolari contenenti la promessa di pagamento di somma certa a giorno fisso, a persona determinata o al portatore, all'ordine o no, purché firmata da un commerciante. 
Tutti coloro che, in quel sistema cambiario, attraverso la tratta, l'accettazione, l'avallo o la girata, sottoscrivessero la cambiale, si consideravono solidalmente garanti ed obbligati al pagamento della somma cambiaria con gli interessi, le spese del protesto ed altre spese legittime, come commissione, bollo e porto, con diritto di regresso fino al traente, per effetto di protesto regolare.

Ciò che caratterizzava e giustificava la tratta della Jettera di cambio o di terra era l'esistenza della provvista dei fondi in potere del trattatario.

5. Non era trascorso mezzo secolo dalla promulgazione del Codice Commerciale del 1850 che si fece sentire, nell'elaborazione dei giuristi, la necessità di una riforma del diritto cambiario.

Nel 1906 veniva presentato alla Camera dei Deputati un progetto destinato a questo scopo. Non si poteva dire fosse veramente una riforma, benché si considerasse che il Codice Commerciale non corrispondeva alle necessità del commercio, e ancora meno allo sviluppo della scienza giuridica. Si proponevano modificazioni al testo legislativo. Alcune delle proposte esprimevano idee della dottrina germanica, ma la cambiale rimaneva legata alla teoria contrattuale.

In quel momento tuttavia un magistrato, allo stesso tempo professore nella Facoltà di Diritto di Minas Gerais, il J. A. Sararva, aveva già pubblicato nella Rivista Forense di Bello Horizonte una serie d'articoli richiamando l'attenzione dei giuristi sulla dottrina cambiaria germanica, articoli riuniti poi in due tomi intitolati Diritto Cambiario Brasiliano.

"La condanna", scrisse il SARAiva, "della dottrina francese, la relazione intima fra l'indole della cambiale e la sua importanza economica, la disciplina dei titoli al portatore sulla base del concetto dell'atto unilaterale nel nostro Codice Civile, lo sviluppo mercantilè ed industriale del paese, la necessità indeclinable e tanto proclamata di 
incrementare attraverso ogni mezzo le relazioni fra piazza e piazza, le modificazioni delle leggi operate per effetto delle decisioni della giurisprudenza in riscontro di imperiose esigenze della vita pratica - queste ed altre circostanze indicano trovarsi il terreno pronto ad accogliere il nuovo istituto cambiario: la strada della riforma é libera".

La coincidenza della pubblicazione di quel trattato di diritto cambiale con la proposta della riforma del capitolo corrispondente del Codice Commerciale, originó una proposizione ampia e sistematica, che sostituiva il progetto in corso alla Camera dei Deputati.

Questa proposta, simile alla legge cambiaria germanica del 24 novembre 1848, nella quale la lettera di cambio era trasformata in semplice strumento di obbligazione, circondata da rigorose formalità estrinseche, libera della necessità di una rimessa di denaro, da luogo a luogo, della valuta somministrata - fu bene accolta e alla fine dei tramiti parlamentari trasformata in legge, attraverso il Decreto n. ${ }^{\circ}$ 2.044, del 31 dicembre $1908^{10}$.

10. DeCreto N. 2.044, 31 Dicembre 1908 - Definisce la lettera di cambio ed il vaglia cambiario e regola le operazioni cambiarie.

Il Presidente della Repubblica degli Stati Uniti del Brasile:

Faccio sapere che il Congresso Nazionale ha decretato ed io sanziono la resoluzione seguente:

\author{
Titolo I \\ Della lettera di cambio \\ Capo I, \\ Della emissione
}

Art. 1. - La lettera di cambio é un'ordine di pagamento e deve contenere per esteso nel contesto questi requisiti:

I, la denominazione "lettera di cambio" o la denominazione consimile nella lingua in cui esso é redatto;

II, la somma di denaro da pagarsi e la specie della moneta;

III, il nome di colui al quale deve farsi il pagamento. Questa indicazione puó essere scritta nel contesto; 
Il processo di rinvigorimento della cambiale convertita in titolo di credito antonomo si é realizzato invero senza le difficoltà che sorgono al momento della trasformazione degli istituti giuridici. Si creó il profilo di un nuovo diritto, che il maestro Lorenzo Mossa ha studiato in Italia come giurista in cerca della verità ed esposto come professore alla cui memoria rendiamo l'omaggio di una fervida ammirazione.

IV, il nome di chi é designato a pagare. La lettera puó essere al portatore o per ordine e conto di un terzo. Il traente puó designarsi come trattario;

$\mathrm{V}$, la sottoscrizione di pugno del traente o di un loro mandatario speciale. La firma deve essere apposta sotto il contesto.

Art. 2. - Non sará lettera di cambio il titolo nel quale manchi alcuni dei requisiti sopra indicati.

Art. 3. - Si considerano questi requisiti come iscritti al tempo dell'emissione della lettera. Sarà ammessa la prova in contrario in caso di mala fede del portatore.

Art. 4. - Si presume il mandato al portatore per inserire la data ed il luogo dell'emissione nella lettera in cui siano omessi.

Art. 5. - In caso di differenza tra il valore in cifra e quello scritto per esteso nel corpo della lettera, quest'ultimo sará sempre considerato come il veritiero, senza danno della lettera. Diversificando le somme di denaro nel contesto, il tijolo nom sarà di cambio.

Art. 6. - La lettera puó essere emessa:

I, a vista;

II, a giorno fisso;

III, a certo tempo vista.

La epoca del pagamento deve essere precisa, una sola per la totalitá della somma cambiaria.

\section{Capo II}

Della girata

Art. 8. - La girata trasferisce la proprietá della lettera di cambio.

La girata é valida ancorché il girante o il suo mandatario speciale scriva soltanto il nome a tergo della lettera.

Il giratario puó completare questa girata.

$\S 1$. La clausola "per procura" apposta sulla girata indica il mandato con tutti $i$ poteri, a meno che vi sia restrizione espressa neila stessa girata. 
6. Innanzi tutto, la legge brasiliana non é la riproduzione testuale della legge germanica: essá si presenta con struttura propria ed in alcuni punti migliorata e quasi portata a quel perfezionamento che il progresso commerciale esigeva.

Cosi ad esempio ha permesso l'emissione della lettera di cambio al portatore; ha fatto della girata, nell'intento di renderlo simile alla moneta, elemento essenziale di un titolo creato e destinato alla circolazione; ha determinato la scadenza anticipata di esso come effetto del protesto

§ 2. La girata posteriore alla scadenza produce gli effetti di una cessione civile.

§ 3. $\mathbf{E}$ vietata la girata parziale.

\section{Capo III}

\section{Dell'accettazione}

Art. 9. - É facoltativa la presentazione per l'accettazione della lettera a tempo certo di scadenza.

La lettera a tempo certo vista deve essere presentata all'accettazione del trattario nel termine stabilito; in mancanza di designazione, entro i sei mesi della data d'emissione, sotto pena di decadere il portatore dai suoi diritti di regresso contro il girante, contro il giratario e contro gli avallanti.

Paragrafo unico - L'accettazione della lettera a certo tempo vista deve essere datata; in difetto si presume al portatore il mandato per datarla.

Art. 10. - Essendo due o piú i trattari, il portatore deve presentare la lettera al primo designato; in mancanza o rifiuto dell'accettazione, al secondo qualora domiciliato nello stesso luogo; e così succesivamente nonostante l'ordine d'indicazione dei nomi dei trattari nella lettera.

Art. 11. - Per la validità dell'accettazione basta la semplice firma autografa del trattario, o del suo mandatario speciale sulla faccia anteriore della lettera.

La dichiarazione che non esprima rifiuto indubitabile, limitazione - modificazione vale come accettazione pura.

Paragrafo unico. - Qualunque limitazione o modificazione equivale a mancanza di accettazione; ma 1 accettante rimane obbligato nei termini della limitazione o modificazione. 
per mancanza o rifiuto dell'accetazzione, nel proposito di non snaturarlo con l'esigenza di guarentigia reale disperdendone la funzione di credito personale in movimento; e finalmente ha equiparato l'avallante al debitore autonomo per evitare l'esistenza, nella cambiale, di vincoli accessori.

Giuridicamente, la lettera di cambio non é piú un contratto di cambio. Mezzo per ottenere e rispettivamente concedere credito, essa é caratteristicamente un titolo di credito all'ordine e perció formale, cioé redatto con forma determinata dalla legge, in difetto della quale non puó avere il valore e gli effetti propri della cambiale.

La lettera di cambio serve con la sua forma a custodire i diritti derivanti da qualsiasi operazione da cui risulti un credito; ed é il documento necessario all'esercizio del diritto letterale che vi é menzionato.

Art. 12. - Una volta prestata, l'accettazione non puó mai essere cancellata o revocata.

Art. 13. - La mancanza o il rifiuto di accettazione si prova mediante il protesto.

\section{Capo IV}

Dell'avallo

Art. 14. Il pagamento di una lettera puó essere garantito cor avallo indipendentemente di accettazione o di girata. Si considera valido l'avallo dato soltanto colla firma del pugno dell'avallante, o del suo mandatario speciale, apposta sulla faccia o a tergo della lettera. e ancora acettata, al traente.

Art. 15. - Si equipara l'avallante a colui il cui nome indica; ma in mancanza di questa indicazione, a quello sotto il cui nome apporta la sua firma; fuori di questi casi, all'accettante, e se la lettera non e ancora accettata, al traente.

\section{Capo V \\ Della moltiplicazione della lettera di cambio \\ Dei duplicati.}

Art. 16. - Il traente, sotto pena di essere tenuto al risarcimento del danno, é obbligato a dare al portatore della lettera le copie da lui richieste prima della scadenza, differenziate nel contesto per numerazione in ordine di seguenza, o riserva di quelle che se siano 
Cosi l'ha concepita la legge brasiliana, che le ha datto un alto valore giuridico per la sua forma letterale e completa: come tale, probat rem ipsam. Essa deve bastare a se stessa. Chi la possiede sa bene l'arma che ha in mano; e chi la firma non puó ignorare che assume una obbligazione certa quanto alla sua esistenza e determinata quanto al suo oggetto: cioé una obbligazione liquida.

Questa obbligazione risponde al diritto astratto di chi l'acquista, indipendentemente dall'affare che diede luogo alla sua emissione. Sono autonome le obbligazioni cambiarie e independenti le une dalle altre: i vizi o deficienze

smarrite. In diffetto di differenziazione o di reserva che esprima l'unicitá dell'obbligazione, si considerano come altretanti cambiali distinte.

$\S$ 1. Il girante ed il suo avallante sono tenuti a riprodurre la tratta e l'avallo nella duplicata sotto pena di risarcimento del danno.

$\S 2$. Il trattario é tenuto responsabile per ciascun degli esemplari nei quali apponga l'accettazione.

$\S 3$. Il traente di due o piú esemplari della lettera a distinte persone ed i successivi traente ed avvallanti rimangono cambialmente obbligati.

$\S$ 4. Il detentore della lettera emessa per l'accettazione é obbligato a consegnarla al legittimo portatore dei duplicati, sotto pena di risarcimento del danno.

\section{Capo VI}

\section{Della scadenza}

Art. 17. - La lettera a vista scade all'atto della presentazione.

La lettera a giorno fisso scade in quel giorno. La lettera a giorno data o dalla vista scade nell'ultimo giorno del termine; ma il giorno della data non é compreso nel termine della prima ed il giorno dell'accettazione nel termine dell'altra.

La lettera a settimane, a mesi o adanni della data o dalla vista scade nel giorno della settimana, mese od anno del pagamento, corrispondente al giorno dell'emissione o dell'accettazione. In mancanza dell'indicazione del giorno corrispondente, scade l'ultimo giorno del mese di pagamento.

Art. 18. - Quando emessa la lettera in paese dove sia vigorante un'altro calendario, senza dichiarazione di quale sia l'adottato, il termine della scandenza si verifica computandosi il giorno del calendario gre- 
che rendono invalida una obbligazione non si comunicano alle altre contenute nello stesso titolo, non pregiudicano la validitá di esso.

La lettera di cambio é un titolo contenente l'obbligazione incondizionata di pagamento di una somma certa: sull'ambigiutá o l'errore della menzione toglie al titolo il carattere di cambiale.

goriano corrispondente al giorno dell'emissione secondo l'altro calen dario.

Art. 19. - Si considera scaduta la lettera protestata:

I, per mancanza o rifiuto di accettazione;

II, per fallimento dell'accettante.

Si riputa differito il pagamento in questi casi al giorno della scadenza ordinaria della lettera, verificandosi l'accettazione dell'altro trattario designato o, in mancanza, il consentimento del portatore all'accettazione per intervento volontario espresso nel atto del protesto.

\section{Capo VII}

\section{Del pagamento}

Art. 20. - La lettera deve essere presentata al trattario o all'accettante per il pagamento nel luogo in essa indicata e nel giorno delia scadenza, oppure essendo quel giorno feriale per la legge, nel primo giorno successivo; in mancanza della presentazione il portatore decade del diritto di regresso contra il traente, il giratorio ed avallante.

$\S 1$. Sará pagabile a vista la lettera senza indicazione dell'epoca di scadenza; e la lettera senza indicazione del luogo di pagamento sará pagabile nel luogo designato sotto il nome del trattario.

E facoltativa l'indicazione alternativa di luoghi di pagamento, assi curato al portatore il diritto d'opzione. La lettera puó esse tratta su di una persona per essere pagata nel domicilio dell'altra indicata dal traente o dall'accettante.

$\S 2$. In caso di rifiuto o mancanza di pagamento dall'accettante, essendovi due o piú trattari, il portatore deve presentare la lettera a! primo nominato, se esso sia domiciliato nella stessa piazza; e così successivamente nonostante la forma d'indicazione nella lettera dei nomi dei trattari.

$\S 3$. In caso fortuito o di forza maggiore la presentazione deve essere fatta dopo la cessazione dell'impedimento.

Art. 21. - La lettera tratta a vista deve essere presentata per il pagamento dentro il termine in essa indicato; e dentro dodici mesi della 
Il rigore formale ha fatto riconoscere un mandato tacito al portatore per completare la data ed il luogo della tratta omessi nel titolo. Così nella legge brasiliana. Invece la legge uniforme dispone che se la lettera di cambio, incompleta nell'atto della emissione, é riempita in contrasto con le convenzioni, l’innosservanza di esse non puó essere opponibile al portatore, a meno che egli abbia acquistato il titolo di mala fede ovvero abbia commesso colpa grave acquistandolo.

data dell'emissione del titolo, in mancanza d'indicazione, sotto pena didecadenza del diritto di regresso contro il traente, il giratario e l'avallante.

Art. 22. - Il possessore della lettera non può essere costretto a ricevere il pagamento prima della scadenza. Chi paga una lettera prima della scadenza é responsabile della validitá del pagamento.

$\S 1$. Il portatore non puó rifiutare un pagamento parziale al tempo della scadenza.

$\S 2$. Il portatore quando ne riceve il pagamento, deve consegnare la lettera quietanzata a chi paga; in caso di pagamento parziale, in cui non si fa la tradizione del titolo, deve farne menzione sul titolo e darne quietanza separata.

Art. 23. - Si presume validamente libero chi paga la lettera alla scadenza, senz'opposizione.

Paragrafo unico. - L'opposizione al pagamento é amessa soltanto in caso di smarrimento della lettera ovvero di fallimento o incapacitá del portatore per riceverlo.

Art. 24. - Il pagamento fatto dall'accettante o dai rispettivi ava!lanti estingue la responsabilitá di tutti i coobbligati; ed il pagamento fatto dal traente, dai giranti ovvero dai rispettivi avallanti estingue la responsabilitá degli coobligati posteriori.

Art. 25. - La lettera di cambio deve essere pagata colla moneta in essa indicata. Se la somma é stabilita in una moneta straniera deve essere il pagamento fatto nella moneta nazionale, al cambio a vista del giorno della scadenza o del luogo del pagamento. Si puó determinare al contrario espressamente sul tijolo. In mancanza del corso del cambio nel luogo, il valore sará determinato secondo il cambio della piazza piú prossima.

Art. 26. - Si la lettera non é presentata per il pagamento alla scadenza, l'accettante, trascorso il termine per fare il protesto, ha diritto di depositare la somma indicata nel titolo a spese e rischio del portatore, senza bisogno di alcuna citazione. 
La legge uniforme ammette implicitamente la convenzione di riempimento della cambiale in bianco fuori dai limiti stabiliti dalla legge brasiliana, piú prudente su questo punto. Si tratta di un contratto verbale che si trasmette identicamente al titolo, da uno all'altro portatore o trattario.

Art. 27 - La mancanza totale o parziale di pagamento si prova mediante il protesto.

\section{Capo VIII}

Del protesto

Art. 28 - La lettera in punto d'essere protestata per mancanza di'accettazione o di pagamento, sará consegnata all'ufficiale competente nel primo giorno sucessivo al rifiuto dell'accettazione o della scadenza ed il rispettivo strumento sará fatto entro tre giorni.

Paragrafo unico. - Il protesto si deve fare nel luogo indicato nella lettera per l'accettazione o per il pagamento.

Tratta o accettata la lettera per pagamento in un altro domicilio che non sia quello del trattario, in questo domicilio deve levarsi il protesto.

Art. 20. - Lo strumento del protesto deve contenere:

I, la data;

II, la trascrizione esatta della lettera e delle dichiarazioni che in essa si trovino, nell'ordine rispettivo;

III, il certificato della notificazione al trattario od all'accettante ovvero agli altri trattari indicati nella lettera per accettarla o pagarla, la risposta avuta o la dichiarazione che non ne fu data alcuna;

Non vi é bisogno della notificazione nel caso che il trattario od l'accettante scriva e firmi sulla lettera la dichiarazione di rifiuto dell'accettazione o del pagamento, ovvero nell'ipotesi del protesto per fallimento dell'accettante.

IV, il certificato di non essere stato trovato o di essere sconosciuta la persona indicata per l'accettazione o il pagamento. In questa ipotesi l'ufficiale deve eseguire la notificazione mediante affissazione dell'atto nei luoghi di stile e mediante publici proclami nei giornali;

V. l'indicazione degli intervenienti e delle firme da essi onorate;

VI, l'accordo del portatore all'accettazione per onore;

VII, la sottoscrizione, sotto sigillo publico, del'ufficiale del protesto.

Paragrafo unico - Lo istrumento del protesto, con la trascrizione nel libro proprio, dev'essere consegnato al portatore della lettera o a colui che abbia fatto il pagamento. 
La legge uniforme si riferisce, nell'edizione francese, "aux accords intervenus"; ma queste convenzioni non si possono opporre al portatore, acquirente in buona fede e senza colpa grave del titolo già riempito.

Art. 30. - II portatore della lettera deve dare aviso al suo girante del marcato pagamento entro due giorni della data del protesto; ed ogni giratario, nei due giorni di ricevimento dell'avviso, al proprio girante, sotto pena di essere tenuto al risarcimento del danno.

Non essendo indicato nella girata il domicilio o la residenza del girante, l'avviso dovrá essere trasmesso al girante precedente che abbia compiuto quella formalitá.

Paragrafo unico. - L'avviso puó eseguirse a mezzo di lettera racommandata. In tal caso la lettera contenente l'avviso sará portata all'ufficio postale affinché sia speduta al destinatario in pacco racommandato.

Art. 31. - In caso di rifiuto di rinvio della lettera da parte da colui che l'abbia ricevuto per firmare l'accettazione o pararla, il protesto puó essere levato mediante un alto esemplare o, in mancanza, secondo l'indicazione del protestante.

Paragrafo unico. - Comprovato il fatto puó essere decretato l'ar.resto del detentore della lettera, salvo consegna della somma cambiaria e per il rimborso delle spese.

Art. 32. - Il portatore che non realizza il protesto in tempo utile e forma regolare decade dal diritto di esercitare il regresso contro il traente il girante e gli avallanti.

Art. 33. - L'ufficiale che non esegui in tempo e forma regolare l'istrumento del protesto, nondimeno d'incorrere nella pena stabilita nel Codice Penale, é tenuto al risarcimento del danno.

$$
\text { Capo IX }
$$

\section{Del intervento}

Art. 34. - Nell'atto del protesto per mancanza o rifiuto dell'accettazione, la lettera puó essere accettata da un terzo con il consenso del portatore.

La responsabilitá di questo interveniente s'equipara alla responsabilitá del trattario che accetta.

Art. 35. - Nell'atto del protesto, eccettuata l'ipotesi dell'articolo precedente, qualunque ha il diritto d'intervenire per pagare la lettera in onore di qualsiasi firma.

$\S 1$. Il pagamento per onore della firma dell'accettante o dei rispettivi avallanti estingue la responsabilitá cambiaria di tutti gli obbligati. 
Dal testo della legge cambiaria italiana sorge, nel pensiero dell'Angeloni, un "diritto patrimoniale". Il diritto di completare, egli ha detto, "é un diritto inerente al titolo, che risulta del titolo e circola con esso, trasferendosi dal prenditore ad ogni portatore successivo. Si tratta di un diritto patrimoniale, che entra irrevocabilmente insieme col titolo, nel patrimonio del prenditore al momento dell'

$\S 2$. Se non é indicata la firma per onore della quale ha luogo l'accettazione, questa si riputa data per onore del traente; ma nella lettera accettata si considera onorato l'accettante.

$\S$ 3. Se piú persone, giá obbligate o no, offrono il pagamento per intervento, é preferita quella il cui pagamento libera il maggior numero di obbligati.

In caso d'intervento per la stessa firma é preferito l'interveniente obbligato; in mancanza di questo, il trattario; in difetto di ambe due l'opzione appartiene al detentore o portatore.

E vietato l'intervento all'accettante ovvero al rispettivo avallante.

\section{Capo X}

\section{Dell'ammortamento}

Art. 36. - In caso di smarrimento, sottrazione o distruzione totale o parziale della lettera, il proprietario, indicando il suo esatto tenore, puó chiedere al giudice competente del luogo di pagamento la notificazione al traente, all'accettante e agli altri coobbligati a non pagarla; e la citazione del detentore a presentarla in giudizio entro tre mesi $e$, in case di smarrimento o distruzione, la citazione ai cobligati ad offrire opposizione, nelle stesso termine, fondata sul difetto di forma del titolo o sulla mancanza di requisito essenziale al esercizio della azione cambiaria.

Queste notificazioni e citazioni debbono essere inserite sul giornale dello Stato e sul Giornale Ufficiale del Distretto Federale e sui giornali indicati dal giudice, oltre l'affissazione nei luoghi di stile e nella Borsa della piazza del pagamento.

$\S 1$. Il termine di tre mesi decorre dal giorno della scadenza; si giá scaduta la lettera, dal giorno in cui si pubblica l'avviso nel giornale ufficiale.

$\S 2$. Nel corso di quel termine, il proprietario, in possessione del certificato della domanda e dell'autorizzazione del giudice, puó esercitare tutti gli atti che tendono a conservare il diritto di credito e, sca- 
emissione della cambiale, e quindi nel patrimonio di ciascun portatore successivo: per cui il sopravvenire della morte o del fallimento del traente (o dell'emitente) nontoglie la leggitimità del riempimento neppure se questo avvenga con l'apposizione di una data di emissione successiva a tali eventi" ${ }^{11}$.

duta la lettera, puó esigere dall'accettante il deposito in giudizio della somma dovuta.

$\S$ 3. Trascorso il termine senza presentazione del portatore leggimato (art. 39) della lettera, o senza opposizione del coobbligato (art. 36 ), il giudice emette un decreto con il quale pronunzia l'ammortamento del titolo smarrito o distruito, autorizzando in favore del proprietario il pagamento della somma depositata, se il deposito sia stato fatto.

$\S$ 4. Per effetto di questa sentenza il proprietario puó esercitare l'azione cambiaria contro l'accettante e gli obbligati.

$\S 5$. Presentata la lettera dal portatore legittimato (art. 39) o fatta l'opposizione dall'obbligato, il giudice decretará pregiudicata la domanda d'ammortamento della lettera, facoltando peró all'interessato il ricorso all'azione ordinaria.

$\S 6$. La sentenza puó essere impugnata con ricorso speciale.

$\S$ 7. Il procedimento d'ammortamento non esclude il ricorso alla duplicata della lettera e neppure dispensa l'avviso immediato dello smarrimento per lettera racommandata al trattario, all'accettante ed agli altri obbligati, nella forma indicata nel paragrafo unico dell'art 30, agli effetti della responsabilitá civile degli altri obbligati.

\section{Capo XI \\ Della rivalsa}

Art. 37. - Il portatore della lettera protestata puó rimborsarsi della somma che gli é dovuta con una nuova tratta a vista su qualsiasi degli obbligati.

Chi ha pagato la rivalsa puó rimborsari nel modo stesso verso gli obbligati anteriore.

Paragrafo único. - La rivalsa deve essere accompagnata della lettera protestata, del istrumento del protesto e del conto di ritorno.

Art. 38. - Il conto di ritorno deve indicare:

I, la somma cambiaria e quella degli interessi legali dal giorno della scadenza;

11. Vittorio ANgeloni, La cambiale e il vaglia cambiario secondo la legge uniforme di Ginevra, Roma, $2^{\circ}$ ed. Ateneo, n. 58, p. 97. 
Questa conclusione non corrisponde al testo della legge brasiliana, per la quale il portatore ha un semplice mandato di riempimento; ed il mandato si estingue per la morte $o$ il fallimento del mandante. La tesi della esistenza di un dirito patrimoniale non può accogliersi senza riserva.

Il, la somma delle spese legali, protesto, commissione, bollo, porto di lettere ed interessi, dal giorno dal quale siano state fatte;

III, il nome della persona sulla quale la rivalsa é tratta;

IV. il prezzo del ricambio, certificato da un corretore o, in diffetto, da due commercianti.

$\S 1$. Il ricambio si regola secondo il corso del cambio della piazza di pagamento sulla piazza del domicilio o residenza delle persone sulle quale é trata la rivalsa; il ricambio dovuto al traente o all'avallante che ha pagato la letera si regola secondo il corso del cambio della piazza in cui é tratta la rivalsa sulla piazza di residenza o del domicilio della persona sulla quale é tratta.

$\S 2$. facoltato l'accumulo dei ricambi nella rivalsa successiva.

\section{Capo XII}

Dei diritti e delle obbligazioni cambiarie

\section{Sezione I \\ Dei diritti}

Art. 39. - Il possessore é considerato proprietario legittimo della !ettera al portatore e della lettera in bianco.

$\mathrm{Si}$ riputa l'ultimo trattario proprietario leggitimo della lettera girata in pieno se la prima girata é sottoscritta dal prenditore e ciascuna delle altre girate dal giratario della girata precedente.

Seguendosi alla girata in bianco un'altra girata, si presume che il girante di questa abbia acquistato per quella girata la proprietá della lettera.

$\S 1$. In caso di pluralitá di prenditori o di giratari, congiunti o disgiunti, colui che sia in possessione della lettera si considera il creditore unico della obbligazione agli effetti cambiari.

$\S 2$. Soltanto in caso di mala fede nell'acquisizione il possessore legittimo secondo questo articolo puó essere tenuto a rinuciare alla lettera di cambio. 
7 Pure non corrispondente anche alla legge brasiliana é la clausola "non all'ordine" od altra equivalente. Essa considera non scritta, per gli effetti cambiari, la clausola che vieta la girata o il prodotto, l'escludente da responsabilitá per le spese e qualsiasi altre dispensate l'osservanza di termini o delle formalitá legale.

Art. 40. - Chi paga non é tenuto ad indagare l'autenticitá delle girate.

Paragrafo unico. - L'interveniente volontaris che paga é surrogato in tutti $\mathrm{i}$ diritti di colui per onore del quale ha pagato.

Art. 41. - Il detentore, benché senza titolo alcuno, si riputa autorizzato a praticare gli atti necessari alla garanzia del credito, ad esigere l'accettazione, levare il protesto ed esigere, all'època della scadenza, il deposito della somma cambiaria.

\section{Sezione II}

\section{Delle obbligazioni}

Art. 42. - Puó obbligarsi cambiariamente chi abbia capacitá civile o commerciale.

Paragrafo unico. - Lo straniero capace secondo la legge brasiliana é ritenuto obbligato per la dichiarazione da lui firmata anche se incapace secondo la sua legge nazionale.

Art. 43. - Le obbligazioni cambiarie sono autonome ossia indepenti le une dalle altre. Il sottoscrittore della dichiarazione cambiaria rimane da essa vincolato e solidalmente responsabile per l'accettazione e per il pagamento, nonostante la falsitá, la falsificazione o la nullitá da qualsiasi altra firma in essa apposta.

Art. 44. - Si considerano non scritte per gli effetti cambiari:

I, la clausola d'interessi;

II, la clausola di proibizione della girata o del protesto, l'escludente da responsabilitá per le spese e qualsiasi altra dispensate l'osservanza di termine o delle formalitá da questa legge;

III, la clausola di divieto della presentazione della lettera all'accettazione del trattario;

IV, la clausola escludente o restritiva della responsabilitá e qualsiasi altra in benefizio del debitore o del creditore fuori dei limiti stabiliti da questa legge.

$\S$ 1. La girata o l'avallo cancellato si considerano non scritti agli effetti cambiari. 
La clausola apposta alla girata com le menzioni "valuta in garanzia", "valuta in pegno" od ogni altra che impliche un pegno, é ignorata dalla legge brasiliana, al contrario di quanto accade nella legge uniforme, nella quale il portatore puó esercitare tutti i diritti derivanti della lettera di cambio; in questa ipotesi, una girata da lui apposta nel titolo é valida soltanto come girata per procura.

8. La legge uniforme poi si é assai differenziata dalla legge brasiliana nel capitolo dell'accettazione: quella legge é particolareggiata, a incominciare dalla possibilità di fissazione o non di termine per la presentazione della

$\S 2$. Non é lettera di cambio il titolo nel quale il traente esclude o restringe la sua responsabilitá cambiaria.

Art. 45. - Con l'accettazione il trattario si obbliga di pagare al traente e rispettivi avallanti.

$\S$ 1. La lettera girata all'accettante puó essere da questo girata ulteriormente.

$\S 2$. Per nuovo giro della lettera girata al traente, al girante o all'avallante rimangano obbligati codebitori intermedi.

Art. 46. - Chi sottoscrive la dichiarazione cambiaria quale mandatario o rappresentante legale di una persona per la quale non ha potere di agire, é obbligato, come se avesse firmato in propria.

Art. 47. - La sostanza, gli effetti, la forma estrinseca ed i mezzi di prova della obbligazione cambiaria se regolano con la legge del luogo dove l'obbligazione fu firmata.

Art. 48. - Qualora liberata della responsabilitá cambiaria, il traente o l'accettante é obbligato a restituire al portatore con l'interessi legali la somma di cui si sia arricchito ingiustamente a suo danno.

L'azione del portatore per questo scopo é l'ordinaria.

\section{Capo XIII \\ Dell'azione cambiaria}

Art. 49. - E esecutiva l'azione cambiaria.

Per questa azione il creditore ha anche il diritto di richiedere la somma che gli sarebbe dovuta per la rivalsa (art. 38).

Art. 50. - L'azione cambiaria puó essere esercitata contra ono, alcuni o tutti gli obbligati, senza osservanza dell'ordine delle girate. 
lettera di cambio al trattario per accettarla. Ma c'é in piú la facoltà conferita al traente di prescrivere che la cambiale sia presentata a termine fisso. Egli puó anche vietare nel titolo che esso sia presentato per l'accettazione, a meno che non sia pagabile presso un terzo, o in luogo diverso da quello del domicilio del trattario, a certo tempo vista. Ogni girante puó prescrivere che la cambiale sia presentata per l'accettazione fissando o non fissando un termine, salvo che il traente l'abbia dichiarata non accettabile.

Art. 15. - Nell'azione cambiaria il debitore puó oppore al portatore soltanto le eccezzioni fondate sui rapporti personali, sui difetti di forma del titolo e sulla mancanza di requisito necessario all'esercizio dell'azione.

Titolo II

Del vaglia cambiario

\section{Capo I, \\ Dell'emissione}

Art. 54. - Il vaglia cambiario ("nota promissoria") é una promessa di pagamento e deve contenere questi requisiti per esteso nel contesto:

I, la denominazione "nota promissoria" o espressione consimile nella lingua in cui essa é redatta;

II, la somma di denaro a pagarsi;

III, il nome di colui al quale deve farsi il pagamento;

IV, la firma autografa dell'emittente o del suo mandatario speciale.

$\S$ 1. Si presume il mandato al portatore di inserire la data e il luogo dell'emissione nel titolo in cui siano state omessi.

$\S 2$. Il vaglia cambiario senza indicazione di scadenza si considera pagabile a vista. In mancanza d'indicazione del luogo di pagamento sará pagabile nel domicilio dell'emittente.

§ 3. In caso di differenza della somma di denaro sará considerata veritiera quella scritta per esteso, nel testo. Diversificando nél contesto l'indicazione della somma in denaro, non sará il titolo vaglia cambiario. 
Invece la cambiale dichiarata non accettabile non è lettera di cambio nel sistema della legge brasiliana, che vieta la clausola proibitiva di presentazione di essa per l'accettazione.

Il trattario puó chiedere, secondo la legge uniforme, che gli sia fatta una seconda presentazione il giorno seguente alla prima, per l'accettazione; e gli interessati non possono valersi dell'innosservanza di tale richiesta se non sia stata menzionata nel protesto.

Su questo punto la legge brasiliana non si pronunzia, ma in realtá esclude questa seconda presentazione, perché impone la consegna della cambiale all'ufficiale nel primo giorno utile seguente al rifiuto dell'accettazione, affinché sia fatto il protesto necessario.

$\S$ 4. Non sará vaglia cambiario lo scritto nel quale manchi alcuno dei requisiti essenziali sopra enumerati. Questi requisiti si considerano apposti al tempo dell'emissione del vaglia cambiario. $\mathrm{E}$ ammessa da prova in contrario in caso di mala fede.

Art. 55. - Il vaglia cambiario puó essere emesso:

I, a vista;

II, a giorno fisso;

III, a certo tempo data.

Paragrafo unico. - L'epoca del pagamento deve essere precisa, una sola per la totalità del debito.

\section{Capo II \\ Disposizioni generali}

Art. 56. - Sono applicabili al vaglia cambiario, con le modificazioni necessarie, tutti le disposizioni del Titolo I di questa legge, eccetto i relativi all'accettazione e ai duplicati.

L'emittente é obbligato nello stesso modo dell'accettante di una lettera di cambio.

Art. 57. - Si rivocano tutti gli articoli del Titolo XVI del Codice Commerciale ed altre disposizioni in contrario.

Rio de Janeiro, 31 Dicembre 1908, 20 della Republica.

Afonso Augusto Moreira Pena

David Campista." 
La legge uniforme non obbliga il portatore a consegnare al trattario la lettera di cambio che gli sia presentata per l'accettazione, e così pure la legge brasiliana. Ma questa ha previsto la consegna del titolo col disporre che, rifiutata la sua restituzione, il protesto puó essere fatto con un altro esemplare $o$, in sua mancanza, mediante l'indicazione del protestante, senza pregiudizio della di lui responsabilità penale.

9. In caso di alterazioni nel testo della lettera di cambio la legge uniforme dispone che chi abbia firmato dopo le alterazioni risponde nei termini del testo alterato; ma chi l'abbia sottoscritto prima, risponde nei termini del testo genuino.

Non dice nulla tuttavia quella legge circa la natura di quelle alterazioni; ma essa, come hanno osservato il Arminjon ed il Carry, ha riunito sotto il nome di "alterazioni" tutte le modificazioni apportate al contesto di una lettera di cambio, volontarie o no, tanto nel testo quanto nella dichiarazione cambiaria per esempio l'aggiunta di un domicilio, la cancellatura di una clausola "senza garanzia", la modificazione del nome del trattario, ecc. L'alterazione puó consistere in una mutilazione, una soppressione mediante lavatura chimica, ecc. ${ }^{12}$.

La legge brasiliana ha fatto una distinzione degna di nota. Posto il principio dell'indipendenza e dell'autonomia delle obbligazioni cambiarie, ha prescritto che il sottoscritore della dichiarazione cambiaria resta vincolato in solido per l'accettazione ed il pagamento della lettera, nonostante la falsità, la falsificazione o la nullità di quanlunque altra firma.

Essa non contiene disposizioni circa le alterazioni del contesto della lettera di cambio, nemmeno nei termini generali della legge uniforme; ma il Codice Commerciale ha stabilito che il documento in cui vi sia cancellatura o

12. P. ARMinjon \& P. CARRY, La Lettre de Change et le Billet. à Ordre, Paris, 1938, Librairie Dalloz, p. 247, n. 221. 
modifica essenziale senza ratifica dei contraenti, sottoscritta da questi, non produce nessun effetto in giudizio; salva la prova di essere stato viziato dalla parte interessata all'invalidità del documento.

Essendo requisiti essenziali della lettera di cambio quelli enumerati dalla legge, in mancanza dei quali il titolo non vale come cambiale, le alterazioni senza ratifiche espresse degli obbligati, da essi sottoscritte, snaturano il titolo. La cambiale dev'essere sopratutto liquida e certa. Così hanno deciso i tribunali brasiliani: la lettera di cambio deve presentarsi in giudizio rivestita di tutte $i$ suoi requisiti essenziali, senza correzioni, cancallature o mutilazioni che la facciano sospetta o incerta ${ }^{13}$.

10. Si potrebbero moltiplicare questi confronti fra le due leggi cambiarie, la uniforme e la brasiliana; ma sarebbe necessario farne un trattato, tanti sono i problemi sorgenti della lettera di cambio nella sua pratica.

Molto si parla del suo formalismo, della sua letteralità, dell'astratezza ed indipendenza delle obbligazioni emergenti del suo contesto sintetizzabili in una sola frase: "Per questa lettera di cambio pagherette a Caio il 31 dicembre 1958 la somma di centomila lire", anteposta la data, indicato il trattario ed il suo domicilio e sottoposta la firma del traente. Ecco il modello classico del titolo nella sua semplicità organica e fisionomica.

Questa frase corta, incisiva, che suona come un commando, perché contiene un ordine di pagamento, é la fonte incessante, ricca di problemi dottrinari e di controversie fra $i$ giuristi ed $i$ tribunali, di provvedimenti del legislatore e delle convenzioni internazionali per ottenere una legge uniforme sulla cambiale.

Si sono realizzate queste convenzioni ed il Brasile ha partecipato attivamente tanto a quella tenuta all'Aia nel

13. Revista dos Tribunais, São Paulo, v. 159, p. 701; v. 161, p. 750 , v. 165, p. 207. 
1912, quanto a quella di Ginevra nel 1930, sotto gli auspici della Società delle Nazioni; ma l'aspirazione era molto alta a poter esser integralmente attenuta.

La Convenzione di Ginevra non ha raggiunto un risultato completo poiché non aveva raccolto che l'adesione di ventisei Stati, mancanti fra gli altri gli Stati Uniti di America e l'Inghilterra. Questi paesi formano così tuttoggi un gruppo ed un sistema giuridico tradizionale. Il mondo non é ancora uno solo, nonostante sia geograficamente contenuto nella unità cosmica della medesima sfera.

Si che - lo scrisse il Rotondi nella prefazione alla sostanziosa monografia del Grisoli su La Cambiale in Diritto Inglese - "si che, dal punto di vista del diritto cambiario, due grandi sistemi si contrappongono ormai, abbracianti i paesi piú numerosi e certo economicamente piú progrediti. E se ad un piú largo risultato la conferenza di Ginevra non é arrivata, é proprio - a giudizio di molti - perché un altro grande sistema unitario si era in sostanza formato ormai nel grande mondo anglo-germanico sulla base della legge cambiaria inglese".

La domanda lanciata dal RoтоNDI, "se sia realizzabili una superiore fusione dei due sistemi con la creazione di un titolo cambiario universale, i cui benefici dal punto di vista pratico non richiedono dimostrazione", rimarrá senza risposta per molti anni ancora.

11. Il Brasile ha firmato la Convenzione di Ginevra, come quella dell'Aia; ma la ratifica di questa convenzione ha posto in linea di esame un problema di ordine costituzionale interno.

Presentato dal Presidente della Repubblica al Congresso Nazionale la Convenzione dell'Aia, per ottenere la necessaria omologazione, la Commissione di Diplomazia e Trattati della Camera dei Deputati si espressò così:

"Il compito di legiferare sul diritto commerciale della Reppublica é prerogativa del Congresso Nazionale, ai termini del art. 34, n. 23, della Costituzione; e non é possibile confonderla con quella di decidere definitivamente sopra 
trattati e convenzioni internazionali con le nazioni esteri a cui si riferisce il $n .12$ dello stesso articolo.

"I trattati regolano le questioni di diritto pubblico e sono stipulati dal Presidente della Repubblica; le leggi e risoluzioni al contrario sono di diritto pubblico interno, hanno la loro volidità dipendente della rigorosa osservanza di un procedimento speciale di elaborazione prescrito in tutte i Costituzioni ed emanano della sovranità nazionale, attraverso l'intermediazione di rappresentanti diretti investiti di poteri idelegabili. Si la Convenzione in esame é veramente un trattato di diritto pubblico esterno, il regolamento uniforme con esso integrato é, a rigore, una legge, di quelle che debbono essere elaborate in conformità col diritto pubblico interno.

"In questi termini, il trattato-legge solamente potrebbe essere possibile senza collisione dei principi di diritto internazionale con i principi di dixitto costituzionale, se il Presidente della Repubblica avesse l'attribuzione di fare delle leggi, alli infuori dei trattati, o se il Potere Legislativo avesse quelle di stipulare i trattati".

"Non si potrebbe pure mettere in vigore la legge uniforme soltanto con l'omologazione dalla quale é risultata. Il Congresso Nazionale avrebbe, in tale caso, delegato in favore di un'assemblea, cui il rappresentante brasiliano ha preso parte, insieme con gli di vari altri paesi esteri, una delle sue prerogative essenziali, mentre al contrario gli'é vietata la delegazione dei suoi poteri in favore di chichessia".

La congrua soluzione sarebbe quella della presentazione del regolamento uniforme come progetto di legge, nella forma prevista dalla Costituzione, affinché si potesse transformare in legge nazionale.

Il Decreto n. 3.766, del 27 agosto 1919, ha approvato la Convenzione Internazionale per l'Unificazione del Diritto Cambiario dell'Aia del 23 giugno 1912; ma ha disposto que la ratifica di quell'atto da parte del Governo sarebbe avvenuta soltanto dopo la conversione in legge di quella Convenzione. 
Per la stessa ragione, la Convenzione di Ginevra non ha forza di legge in Brasile; ma la legge cambiaria brasiliana del 1908 é la piú prossima alla legge uniforme di Ginevra ed ha la virtù di averla antecipata di molti anni.

12. La Costituzione di Francia del 1946 ha aperto la possibilità della soluzione per via diplomatica del problema dell'unificazione delle leggi uniformi di diritto privato.

L'importanza del nuovo dispositivo costituzionale francese é considerabile: per esso, "les traités diplomatiques regulièrement ratifiées et publiés ont force de loi dans le cas même óu ils seraient contraires à des lois internes françaises, sans qu'il soit besoin pour en assurer l'application d'autres dispositions legislatives que celles qui auraient été nécessaires pour assurer leur ratification".

Questi trattati, "ayant une autorité supérieure à celles des lois internes, leurs dispositions ne peuvent être abrogées, modifiées ou suspendues qu'a la suite d'une dénonciation régulière, notifiée par voie diplomatique".

La Costituzione brasiliana del 1946 non contiene identica disposizione.

Per la Convenzione di Ginevra gli Stati contraenti si impegnarono ad introdurre nei loro territori la legge uniforme tal quale é stata stabilita; ma nel territorio brasiliano non si ha fatto sentire la necessità dell'introduzione di quella legge perché esisteva già, ed é ancora in vigore, una legge elaborata per ispirazione dei medesimi principi di ordine economico e giuridico. 